ment, needles and thread, we think this danger would be eliminated.

The next question would be, shall we get adhesions by holding the uterus in contact with the abdominal wall in this way? We think we should, because by pulling the uterus up against the abdominal wall, these stitch holes must naturally give a little, perhaps they will even tear some; this will set up some local inflammatory action which will result in adhesions, which "report of cases" will prove, if the nterus is brought in firm contact with the abdominal wall. We may either leave this stitch in there permanently or may remove it after two to three weeks.

The only contra-indication for this operation, as far as we can see, would be adhesions of the intestines to the fundus uteri or portions of the abdominal wall, or firm adhesions of ovaries and tubes to the back, or presence of pus about the uterus and appendages. The interference with a large bladder can be easily avoided by keeping a sound in it during the operation.

Whoever has operated or seen the operation, will be convinced that the danger of wounding the intestines is almost eliminated because the uterus can be so plainly felt through the abdominal wall and manipulated in such a manner that all intervening tissues can positively be excluded.

Case 1.-Miss M. D., age 19. Retroversion with strong adhesions, after severe fall four years ago; since that time frequent painful urination, backache and difficult menstru-
ation. I made the first operation at Bethany Hospital. Kansas City, Kan., April 21, under assistance of Drs. J. D. Griffith and S. I. Harrison, and R. A. Roberts, of Kansas City. Uninterrupted recovery; highest temperature recorded was 99.4. Patient had no pain nor inconvenience whatever and was discharged after three weeks'stay, (two weeks in bed) in the Hospital, with her uterus firmly adherent to abdominal wall hardly within the reach of the finger in vagina.

Case 2.-Mrs. F. O., age 37. Three children, youngest 5 years old; after last confinement, prolapsus uteri with pertaining complaints; operated April 22 ; highest temperature 99.7; very little pain; remained in bed thirteen days; discharged after three weeks; uterus in proper place. Uterine supporter was fitted to be worn about one or two months.

Case 3.-Mrs. R. T., age 26. After tedious labor case and forceps delivery, patient complained of backache, headache, and frequent painful urination. Examination showed retroflexio uteri. Operation April 24; temperature remained normal; slight pains over region of stitch. Contrary to my custom I did not embed the stitch in abdominal tissue but severed it the seventh day, in order to test adhesions which proved strong enough to make the uterus adhere to the abdominal wall. Uterine supporter was fitted and patient was discharged seventeen days after operation completely cured.

Case 4.-Mrs. A. K., age 43. Prolapsus uteri of nine years' standing. Operated April 25 ; clipped stitch May 2 ; found uterus strongly adherent and fitted uterine supporter. Discharged May 19 completely relieved.

Case 5-Miss F. L., age 18 years. Kicked by horse in the abdomen about one year ago; since that time dysmenorrhea, headache, backache, fatigue feeling, etc. Diagnosis, retroflexio uteri. Operated April 26. No elevation of temperature; had a circumscribed peritonitis around the stitch as large two silver dollars. Made uninterrupted recovery and was discharged two weeks after the operation.

Case 6.-Mrs. A. B., age 25 . Gave birth to twins, forceps delivery, two years ago. Diagnosis, retroflexio uteri. Was unable to do her own housework because of backache, headache, and constant desire to urinate. Operated April 29; highest temperature 99 ; very little pain. Did not embed stitch but cut it twelve days after operation; uterus remained in place. Uterine supporter fitted and patient was discharged twenty days after operation completely relieved.

Case 7.--Mrs. L. M., age 36. After fall from a wagon two years ago had adhesive retroversio uteri ; difficult menstruation with constant backache and headache. Operated May
1 ; no rise of temperature; was discharged relieved, within two weeks after operation.

Case 8.-Mrs. J. K., age 26. Had three children. According to her own report had miscarriage two years ago; after that puerperal fever. Examination shows retroverted uterus almost immovable. Operation May 4; greatest difficulty in destroying adhesions. Rise of temperature second day to. 102.2 ; third 101 ; the following day 100.3 , but found in changing the vaginal tampon that uterus had dropped back as I expected.

There were three more cases operated upon which gave complete satisfaction though they are still under treatment, therefore reports later.

\section{THE SURGICAL TREATMENT OF GRANULAR CONJUNCTIVITIS.}

BY J. A. BACH, M.D.

PROFESSOR OF DISEASES OF THE EYE AND EAR, WISCONSIN COLLEGE OF PHYSICIANS AND SURGEONS, MILWAUKEE, WIS.

The important feature in all forms of treatment of granulations of the eye-lids, whether surgical or otherwise, is to remove pathologic elements and prevent further invasion of the same with their destructive consequences, with the least possible harm to the conjunctiva and lids. Without proper treatment we know that granulations will in many cases ruin eyes, and in others cause serious lesions, either by inducing ulcerative processes or, in the more favorable cases, by producing cicatricial contractions and deformities of the lids. In fact, a large percentage of cases as they come for treatment, have already suffered in some way or other in the mobility or form of the eye-lids. These complications by the additional irritation they induce, further aggravate the unpleasant symptoms and not infrequen1ly produce spasmodic contractions of the muscles of the lids, forming a serious obstacle to the successful treatment of the case. Therefore, often one of the first considerations in the surgical treatment is to remove such complications by the proper operation. A canthotomy in cases where this is indicated, through the powerful contractions of the orbicularis muscles, will give us the most valuable aid as a preliminary step to further treatment.

Other abnormal conditions as to mobility or shape of the eye-lids can frequently be corrected with great advantage before proceeding far in the treatment proper of the case before us.

The surgical treatment to be of any advantage in the cure of granulated eye-lids must be of a conservative nature and must seek, as far as possible, to protect rather than destroy invaded elements of the conjunctiva and deeper tissues. We must therefore place as foremost of all surgical measures, the expression rather than excision of the pathologic elements in all cases where this can be done. By this means we may not succeed completely in removing all the granulations, but we will succeed in suitable cases if the operation is thoroughly done, as it ought to be, to remove most of them and at the same time so stimulate absorption and physiologic tissue changes as to lead to a rapid cure of the condition, whether supplemented by further local applications or not. It is not to be forgotten that the ordinary remedies are applied, not with the idea of directly destroying the granulations but for the purpose of inducing that state of local hyperemia which most favors absorption, so that if we can directly, by expression remove these deposits, we gain valuable 
time besides producing the same results physiologically, that topical applications produce.

Expression of granulations can be used in all th cases, but is more especially adapted to the first stages of the disease, before the deeper tissues are much involved. Where severe inflammatory symptoms usher in the disease, it is well to allay such inflammations before applying surgical measures of any kind.

A number of inetruments have been devised for the purpose of expression, the most suitable of which are Knapp's roller forceps and Noyes' forceps. Another instrument that can be used with great advantage is the ordinary Desmearre's entropion lid forceps, and which in many cases I prefer to other means. However, it is not the particular instrument that one may use in this performance; it is the completeness with which the operation is done that goes to cure our case. Frequently, expression can be successfully done by the use of the finger nails. Friction, as applied by means of a brush or similar means sufficiently strong to tear the superficial capillaries and soft friable granular masses, but without destroying the normal tissue fibers has been extensively used in some quarters with great satisfaction. Grattage or scratching by means of a stiff brush or by a small metal rake, is a similar means but rather more severe, and ought to be used with care and discrimination. In the first stages of the disease it might do considerable harm by tearing and lacerating the conjunctiva and therefore it is applicable only where we have exuberant and fleshy granulations covering the whole lids; where the conjunctiva is so completely infiltrated and covered as not to be recognizable. Expression in such cases is frequently not sufficient, as we have here to deal largely with hyperplasia of tissue elements. The results obtained by friction or grattage as indicated, will by tearing the superficial capillaries, as well as the softer pathologic elements, cause local depletion and greatly improve circulation and absorption. It is surprising how quickly these thick fleshy lids can be reduced in a few days by this means, where it would take months by means of the ordinary topical stimulation. The good results of this procedure can be augmented by the additional use of antiseptics and astringents. In the more advanced stages of the disease, where deep deposits have taken place especially at and in the fold of transmission, the venous circulation often becomes very sluggish owing to mechanical obstruction. Absorption under such circumstances is deficient and practically at a standstill. Neither expression nor grattage is fully capable of removing this condition of affairs. Thorough massage has produced good results, although this is not always sufficient. Through the thickening and stretching produced, the conjunctiva becomes redundant and the careful excision of the fold of transmission has been practiced in such cases with decidedly satisfactory results.

A two-fold indication is met in this way :

In the first place we remove a large amount of infiltrated, and objectionable tissue, and in the second place we destroy the over dilated and inactive blood vessels and improve the local circulation and nutrition.

It has been held that by means of excision of the fold, disagreeable contractions are liable to occur. This, however, is not the case if the excision is done in the proper cases and properly executed. It is de- sirable to excise as little as possible of the conjunctiva, and as this membrane is quite redundant in these cases no harm can come from this. The main object is to excise the subconjunctival tissue deep in the fold.

Galezowski is an ardent advocate of excision of the fold of transmission, and does this as a routine practice in all advanced cases. In his experience he has had no unpleasant results following the operation. Special instruments have been devised for the purpose of excision, among which may be mentioned Galezowski's forceps.

Cauterization by the actual cautery has been practiced to a limited extent and, in my opinion, can not be considered a rational measure. It certainly offers no advantages over excision or grattage, besides being much more liable to leave hard and irregular cicatrices. The principle of electrolysis which has been practiced by different men with good results, would seem more conservative and therefore more applicable. I have had no experience with this method, nor do I believe that it can be made to answer the purpose as well as other means at our command. The operation of scarification of flabby granulations is in my opinion objectionable, as by its use we will necessarily sever and greatly injure the conjunctival covering without offering any advantage which can not better be achieved by grattage. The use of massage, although not strictly a surgical measure is one of great advantage in conjunction with the various other procedures. In cases where the disease has run its course and has left behind large atrophic areas of the conjunctiva, or where this has occurred through severe caustic or surgical treatment, transplantation of conjunctiva of the rabbit to cover these defects, might suggest itself. By thoroughly removing the overlying tissues, it would seem simple enough to transplant healthy conjunctiva and give the globe a more acceptable protection.

\section{INTRALIGAMENTOUS AND RETROPERY- TONEAL TUMORS OF THE UTERUS AND ITS ADNEXÆ.}

Abstract of a paper read at the meeting of the American Gynecological Association, at Washington, D. C., May 29, 1894 .

BY WILLIAM H. WATHEN, M.D. LOUISVILLE, KY.

While paroöphoron cysts and retroperitoneal myomata of the uterus and its muscular processes have nothing in common in their etiology, I would include both kind of tumors, because the technique of the operation for their successful removal is practically identical. With few exceptions, cysts that unfold the layers of the broad ligament and burrow deep into the pelvis and retroperitoneal connective tissue, often developing nearly continuity in relation to other structures, probably arise from remnants of the mesonephros in the paroöphoron. Either the cystic or solid tumor may separate the peritoneum anteriorly and lie in front of it attached to the bladder, fascia and muscles of the abdominal wall, the separation sometimes being so extensive that it may be possible to reach the tumor and enucleate it through a median incision between the pubes and umbilicus without wounding the peritoneum. The separation, however, is usually from the posterior pelvic and abdominal walls; the tumor having passed beneath Douglas' sac unfolds the mesorectum, meso- 